\title{
BMJ open Governance of preventive Health Intervention and On time Verification of its Efficiency: the GIOVE Study
}

\author{
Francesco Saverio Mennini, ${ }^{1,2}$ Gianluca Baio, ${ }^{3,4}$ Giuseppe Montagano, ${ }^{5}$ \\ Gabriella Cauzillo, ${ }^{5}$ Francesco Locuratolo, ${ }^{5}$ Gerardo Becce, ${ }^{5}$ Lara Gitto, ${ }^{1}$ \\ Andrea Marcellusi, ${ }^{1}$ Peter Zweifel, ${ }^{6}$ Alessandro Capone, ${ }^{2}$ Giampiero Favato ${ }^{2}$
}

To cite: Mennini FS, Baio G, Montagano $\mathrm{G}$, et al. Governance of preventive Health Intervention and On time Verification of its Efficiency: the GIOVE Study. BMJ Open 2012;2:e000736. doi:10.1136/

bmjopen-2011-000736

- Prepublication history for this paper is available online. To view this file please visit the journal online (http://dx. doi.org/10.1136/ bmjopen-2011-000736).

Received 6 December 2011 Accepted 10 February 2012

This final article is available for use under the terms of the Creative Commons Attribution Non-Commercial 2.0 Licence; see http://bmjopen.bmj.com

For numbered affiliations see end of article.

Correspondence to Dr Professor Francesco Saverio Mennini; f.mennini@uniroma2.it

\section{ABSTRACT}

Objectives: The GIOVE Study was aimed to the achievement of allocative efficiency of the budget allocated to the prevention of human papillomavirus (HPV)-induced diseases. An ex-ante determination of the most efficient allocation of resources between screening and multicohort quadrivalent immunisation programmes was followed by the ex-post assessment of the allocative efficiency actually achieved after a 12-month period.

Design: A bound optimisation model was developed to determine the ex-ante allocative efficiency of resources. The alternatives compared were the screening programme alone and the quadrivalent immunisation with access to screening. A sensitivity analysis was carried out to assess the uncertainty associated with the main inputs of the model. Subsequently, a cohort of girls with a complete recorded vaccination history were enrolled in an observational retrospective study for 18 months to ensure full compliance with the recommended schedule of vaccination (0, 2, 6 months) within a 12-month time horizon.

Setting: Basilicata region, in the south of Italy. Participants: 12848 girls aged 12, 15, 18 or 25 years. Intervention: Immunisation with quadrivalent anti-HPV vaccine.

Outcome measures: The vaccination coverage rate was considered to be the indicator of the best achievable benefit, given the budgetary constraints.

Results: Assuming a vaccine price of $€ 100$ per dose, a vaccination coverage rate of $59.6 \%$ was required for the most effective allocation of resources. The optimal rate of coverage was initially in favour of the multicohort strategy of vaccination against HPV $(72.8 \% \pm 2 \%)$. When the price paid for the quadrivalent vaccine dropped to $€ 85$ per dose, the most efficient coverage rate $(69.5 \%)$ shifted closer to the immunisation rate actually achieved during the 12-month observation period.

Conclusions: The bound optimisation model demonstrated to be a useful approach to the ex-ante allocation and the ex-post assessment of the resources allocated to the implementation of a multicohort quadrivalent anti-HPV vaccination programme.

\section{ARTICLE SUMMARY}

Article focus

- At present, screening and immunisation are cost-effective strategies to reduce the death toll of cervical HPV-related malignancies, which in Italy alone amounts to 1200 women per year.

- While there are no evidences to reduce the coverage of screening in favour of a wider coverage of immunisation, the adoption of a multicohort strategy of vaccination would allow a larger proportion of the female population aged between 12 and 25 years to be immunised in a shorter period of time ( $6-8$ years).

- A bound optimisation model was developed to determine the allocative efficiency of the resources used for the screening and the vaccination programmes. Subsequently, an observational retrospective study was carried out to verify the degree of allocation efficiency actually attained after implementation of the multicohort immunisation programme.

Key messages

- Using a vaccine price of $€ 100$ per dose, a coverage rate of $59.6 \%$ was required for the most effective allocation of resources. A sensitivity analysis showed that when the price was reduced to $€ 85$ per dose, the most efficient coverage rate increased to $69.5 \%$. A vaccination coverage rate of $72.8 \% \pm 2.0 \%$ was observed.

- Although statistically significant, the observed inefficiency was progressively reducing from $21 \%$ in the July 2007 to August 2008 period to $5 \%$ after September 2008, when the vaccine price was reduced to $€ 85$ per dose.

- The bound optimisation model demonstrated to be a useful approach to assess the allocative efficiency of the resources budgeted to the implementation of a multicohort quadrivalent anti-HPV vaccination programme.

\section{INTRODUCTION}

Human papillomavirus (HPV)-induced malignancies represent the second most 


\section{ARTICLE SUMMARY}

\section{Strengths and limitations of this study}

- The relevance of this study is enhanced by its internal validity: the sample size was significant (over 12000 girls enrolled) and coherent (all subjects enrolled were coming from the same region); the observation time allowed to cover the entire immunisation cycle; individual data related to vaccination (including all costs incurred) were complete and accurate (provided by the Regional Health Authority); the bound optimisation model adequately described the available competing choices: immunisation versus screening.

- The bound optimisation model is subject to the condition that the programmes evaluated are completely divisible, with constant return to scale and that every subject included receives a fraction of the total expected benefit. Nonetheless, these limitations do not seem to affect the generalisability of the research outcomes.

common type of cancer in women worldwide. ${ }^{1}$ In Italy, more than 3000-3500 new cases of cervical cancer (which corresponds to an age-standardised rate of incidence of between 7.7 and 8.1 cases per 100000 women) are diagnosed annually, ${ }^{2}{ }^{3}$ and approximately 1200 women die from this disease every year. ${ }^{2}$ Overall, the economic burden to the Italian National Health Service that is caused by cervical HPV-related pathologies is considerable, with the cost estimated to lie in the range $€ 200-250$ million per year. ${ }^{45}$

A programme of screening for cervical cancer has been implemented in Italy since 1996 to reduce the incidence of cervical cancer and its associated mortality rate. Women aged between 25 and 64 years are invited for screening for cervical cancer every 3 years, with the aim of achieving the early detection and treatment of precancerous cervical lesions (cervical intraepithelial neoplasia $(\mathrm{CIN})$ ) and preventing the onset of invasive cervical cancer. ${ }^{6}$

In early 2007, a new tool became available to reduce the incidence of HPV-related malignancies: the vaccine in its bivalent and quadrivalent form. The costeffectiveness of immunisation against HPV had been previously demonstrated by a large number of modelling studies. ${ }^{7-13}$ In one such study that was carried out in Italy, the authors assessed the cost-effectiveness of a programme of quadrivalent vaccination for a single cohort of 12-year-old girls. ${ }^{14}$ In their model, the current programme of screening (including the management of HPV-related pathologies) was considered as a comparative case. The results of their study suggested that the introduction of a programme of vaccination using a quadrivalent HPV vaccine alongside the current programme of screening for cervical cancer in Italy would produce an incremental cost-effectiveness ratio of $€ 12303$ per life year gained and $€ 9569$ per qualityadjusted life year gained. An economic assessment of the bivalent vaccine reported a cost of $€ 26361$ per quality-adjusted life year gained, ${ }^{15}$ which provided further evidence that vaccination against HPV is currently significantly below the threshold value of $€ 30000-45000$ that is used commonly to determine 'value for money' in health interventions. ${ }^{16} 17$ The difference in the economic evaluation of the two anti-HPV vaccines were mostly determined by the quadrivalent's efficacy in preventing anogenital warts, a nonlife-threatening HPV-induced disease.

More favourable economic results were obtained using predictive models that were based on a quadrivalent multicohort strategy of vaccination (three to four cohorts). ${ }^{18}$ When a multicohort strategy of vaccination is adopted, a larger proportion of the female population aged between 12 and 25 years can be vaccinated in a shorter period of time (5-8 years) and an early reduction of costs can be expected. A multicohort programme of vaccination using the quadrivalent vaccine was associated with a total saving of approximately $€ 132$ million, as a result of a reduction in the numbers of low-grade and high-grade cervical lesions, anogenital warts and invasive cervical cancers (table 1$){ }^{21}$

In July 2007, a decree issued by the State-Regions Conference granted access to quadrivalent vaccination against HPV, which should mandatorily be both free of charge and promoted actively, at least in the cohort of girls aged 12 years, with the progressive achievement of a rate of coverage of $95 \%$ in the next 5 years. The decree was resolving the uncertainty related to the

Table 1 Projected outcomes averted by means of the quadrivalent vaccine and expected reductions in expenditure

\begin{tabular}{llrr}
\hline Outcomes & $\begin{array}{l}\text { Annual expected } \\
\text { number of events }\end{array}$ & $\begin{array}{l}\text { Number of events prevented } \\
\text { with the quadrivalent vaccine }\end{array}$ & $\begin{array}{c}\text { Reduction in } \\
\text { expenditure (€ million) }\end{array}$ \\
\hline Abnormal pap smears & 415000 & 258337 & 3.8 \\
Colposcopies & 116000 & 76909 & 19.2 \\
LSIL and ASCUS & 91000 & 56648 & 22.7 \\
HSIL & 13700 & 9097 & 8.2 \\
Cervical cancer & 4000 & 2988 & 49.9 \\
Genital warts & 125000 & 115625 & 27.8 \\
Total & 764700 & 519604 & 131.6 \\
\hline In this multicohort model, a vaccination coverage rate of 80\% was assumed. ${ }^{19}$ 20 Reductions were calculated at the peak efficiency of the \\
vaccination programme. A cross-protection effect of the quadrivalent vaccine was also considered. \\
ASCUS, Atypical Squamous Cells of Undetermined Significance; HSIL, High-grade Squamous Intraepithelial Lesions; LSIL, Low-grade \\
Squamous Intraepithelial Lesion.
\end{tabular}


choice of the vaccine (quadrivalent) and the priority of cohort to be immunised (12-year-old girls), but the actual modalities of implementation of the immunisation campaign were delegated to individual regions, under the constraint that the incremental cost of the anti-HPV vaccination would be funded within a budget previously allocated to healthcare. In order to maximise the net benefits or utilities to public health that can be derived from a proposed decision, it is essential to allocate resources as efficiently as possible, in full compliance with the universal principles of equity of access to treatment. ${ }^{22}$

The policy makers of the Basilicata region, in the south of Italy, became concerned about the potential issues of equality and allocative efficiency of resources raised by the choices at hand.

A downsize of the current screening programme could potentially increase the risk of HPV-related malignancies, in the light of the lack of information about the impact of immunisation on the coverage and frequency of screening. In time, women vaccinated in the multicohort immunisation programme would enter the age group 25-64 years and thus should still be considered eligible for screening. The option to reduce the resources dedicated to screening would therefore be unethical and, ultimately, it would be likely to have an adverse effect on the women's welfare.

On the other hand, the implementation of a quadrivalent multicohort strategy of vaccination in the region could provide clinical and economic benefits $5-8$ years earlier than would be expected with the 12-year-old single-cohort strategy made mandatory by the national guidelines. It should be considered, though, that the validity of cost-effective studies is strongly dependent on the accuracy of complex models, based on hundreds of assumptions often derived by sparse sources. In spite of the prescriptive nature of health economic outcomes, they cannot completely resolve the uncertainty at the time of the investment decision, determined by the lack of information on population-wide efficacy of the anti-
HPV vaccine in the long term. Moreover, a multicohort vaccination strategy would increase the complexity of the immunisation programme, whose rate of implementation represents an unknown variable itself in the simpler one-cohort model.

After a number of consultations, the healthcare policy makers of the Basilicata region made the following implementation choices:

- To adopt a four-cohort immunisation strategy targeting women who were born in 1983, 1990, 1993 and 1997. Data on vaccination coverage rates by cohort are reported in table 2 .

- To maintain the coverage rate (women aged between 25 and 64 years) and the frequency (3 years) of the screening programme.

- To allocate a maximum allowable budget for 2007/ 2008 to finance both the screening and the immunisation programmes of $€ 2.5$ million.

These constraints were added ex-ante to the choice of the quadrivalent anti-HPV vaccine and the long-term target coverage for the 12-year-old cohort of girls $(95 \%$ in 5 years), which were mandated by Law (Ministry of Health) at national level.

The objective of this study was to support the healthcare management of the Basilicata region to achieve the allocative efficiency of the allowable budget assigned to anti-HPV screening and quadrivalent immunisation programmes. In general terms, the allocative efficiency of a budget between two healthcare programmes is reached when the population welfare is maximised. ${ }^{23}$ As this particular budget was earmarked to prevent HPVrelated malignancies, its allocative efficiency would be reached when the number of HPV-related events is minimised, given three additional conditions: no change in the frequency and coverage of the current screening programme, a multicohort modality of implementation of the new immunisation programme and the achievement of the target coverage required by the national guidelines for the cohort of 12-year-old girls. A further

Table 2 Data on vaccination rates provided by the Basilicata region and included in the bound optimisation model

\begin{tabular}{|c|c|c|c|c|c|}
\hline & Cohort 1996 & Cohort 1993 & Cohort 1990 & Cohort 1983 & All cohorts \\
\hline Girls eligible for vaccination & 2785 & 3064 & 3426 & 3573 & 12848 \\
\hline Vaccinated in LHA 1 & 414 & 456 & 519 & 272 & 1661 \\
\hline Vaccinated in LHA 2 & 825 & 857 & 873 & 739 & 3294 \\
\hline Vaccinated in LHA 3 & 278 & 295 & 361 & 234 & 1168 \\
\hline Vaccinated in LHA 4 & 502 & 470 & 579 & 370 & 1921 \\
\hline Vaccinated in LHA 5 & 341 & 371 & 344 & 249 & 1305 \\
\hline Total vaccinated & 2360 & 2449 & 2676 & 1864 & 9349 \\
\hline Vaccination coverage rate (\%) & 84.7 & 79.9 & 78.1 & 52.2 & 72.8 \\
\hline SD (\%) & 2.0 & 4.3 & 3.4 & 3.1 & 2.0 \\
\hline
\end{tabular}

Differences in vaccination coverage rates were statistically significant between cohorts $(p<0.0001)$. Overall, the vaccination coverage rate reached $72.8 \% \pm 2.0 \%$ after 12 months. The highest vaccination coverage rate was reported in the cohort of girls aged 12 years $(84.7 \% \pm 2.0 \%)$. However, it should be noted that similar values were recorded in the two chronologically subsequent cohorts (15 and 18 years) with rates of $79.9 \% \pm 4.3 \%$ and $78.1 \% \pm 3.4 \%$ for girls aged 15 and 18 years, respectively. In contrast, a statistically significantly lower coverage rate $(52.1 \% \pm$ $3.1 \%)$ was observed in the cohort of women aged 25 years. This rate was significantly below the average value of $72.8 \%(p<0.0001)$ and was also significantly less than the value required to achieve the allocative efficiency predicted by the bound optimisation model $(p<0.001)$. LHA, Local Health Authority. 
condition required by allocative efficiency is that the allowable budget is entirely spent. To date, no studies have examined specifically the level of allocative efficiency of resources assigned to programmes of vaccination against HPV. In order to provide quantitative guidelines relevant to managerial decision, the main objective of the study was further divided into two complementary outcomes:

1. Objective ex-ante: the determination of the most efficient allocation of resources between the screening and the multicohort quadrivalent immunisation programme, given the constraints discussed above.

2. Objective ex-post: the assessment of the allocative efficiency actually achieved by the screening and the quadrivalent immunisation programmes 12 months after the beginning of its implementation (July 2007).

The research outcomes were strictly dependent upon the given constraints stipulated ex-ante by political choices made at regional level, namely the choice of the quadrivalent anti-HPV vaccine, the multicohort immunisation strategy and the frequency and coverage of screening. It was beyond the scope of this paper to determine and compare the allocative efficiency of alternative scenarios based on different constraints (eg, the choice of the bivalent anti-HPV vaccine, a singlecohort immunisation strategy or a different frequency and coverage of the screening programme). The sensitivity analysis reported in the discussion of the results provides an indication of the sensitivity of allocation efficiency to the main parameters taken into consideration, including the allowable budget, the size of the female population eligible for immunisation and/or screening, the annual cost of immunisation and screening and their relative efficacy in preventing HPVrelated diseases, such as abnormal Pap smears, precancerous cervical lesions (CIN1, CIN2-3), invasive cervical cancer and anogenital warts.

\section{METHODS}

Ex-ante objective: the determination of the allocative efficiency of the allowable budget between screening and quadrivalent multicohort vaccination

The bound optimisation model

The bound optimisation model was adopted in view of the fact that a number of ex-ante constraints could be taken into account in the allocation of resources among healthcare programmes. The bound optimisation model was developed by Weinstein and Zeckhauser ${ }^{24}$ and further modified by Garber and Phelps. ${ }^{25}$ It allows makers of health policy to maximise the total expected benefits for a given budget. We used an adapted version of this model widely used in our previous analyses. ${ }^{19} 26$

The general problem of bound optimisation proposed in this study can be described as follows: we wish to choose $\delta_{\mathrm{I}}$ to maximise.

$$
\mathrm{J}=\sum_{\mathrm{i}=1}^{\mathrm{M}} \delta_{\mathrm{i}} \mathrm{x}_{\mathrm{i}}^{1}
$$

subject to constraint $\sum_{\mathrm{i}=1}^{\mathrm{M}} \delta_{\mathrm{i}} \mathrm{x}_{\mathrm{i}}^{2} \leq \mathrm{R}$ and

$$
0 \leq \delta_{\mathrm{i}} \leq 1(\mathrm{i}=1, \ldots, \mathrm{M})
$$

In (1), $x_{i}^{1}$ is the consequential expected benefit from every alternative i. $\delta_{\mathrm{i}}$ represents, instead, the fraction of every alternative that should be chosen. The constraints point out as the amount of the costs $x_{i}^{2}$ associated to the select alternatives must be smaller or equal to the available general budget $\mathrm{R}$. The optimal values of $\delta_{\mathrm{i}}$ are data from a resolution from the equation of this form:

$$
\delta_{i}^{*}= \begin{cases}1 \quad & \text { if } \frac{x_{1}^{1}}{x_{1}^{2}}>\lambda, \\ 0 \quad & \text { if } \frac{x_{i}^{1}}{x_{1}^{2}}<\lambda, \\ \pi & \text { if } \frac{x_{i}^{1}}{x_{1}^{2}}=\lambda, 0<\pi<1\end{cases}
$$

where $\lambda$ is defined as the critical ratio (ie, the optimal proportion for every of the considered alternatives) of the problem of bound optimisation.

\section{Formulation of the problem}

In the model that we adapted, $\mathrm{x}_{1}$ represents screening, $\mathrm{x}_{2}$ is the combination of vaccination and screening and the parameter $\mathrm{E}_{\mathrm{i}}(\mathrm{i}=1,2)$ denotes the expected benefit of each alternative. $\mathrm{B}$ is the budget, and $\lambda$ and $\mu$ are the so-called critical values (ie, the optimal proportions of the alternatives considered). The vaccination coverage rate was considered to be an indicator of the best achievable benefit, given the budgetary constraints in Basilicata. The alternatives compared in the study were as follows:

- The screening programme $\left(\mathrm{x}_{1}\right)$ for the early detection of cervical cancer (ie, secondary prevention). In Italy, organised screening is based on the Pap smear test, which is currently recommended to be carried out every 3 years;

- The quadrivalent programme of vaccination $\left(\mathrm{x}_{2}\right)$ associated with access to screening for cervical cancer for the four cohorts of women eligible to be included in the multicohort vaccination programme. In the present context, the bound optimisation model requires the following data:

- Annual cost of each alternative. Only annual direct costs were included in the analysis due to the lack of data on indirect costs associated with the prevention of HPV-related malignancies in Italy. The annual cost of screening $\left(\mathrm{C}_{1}\right)$ includes the cost of outpatient visits and the materials used to perform the Pap smear test. The combined annual cost of vaccination and screening $\left(\mathrm{C}_{1}+\mathrm{C}_{2}\right)$ also includes the cost of the vaccine.

- Annual budget available for the primary and secondary prevention of HPV-related diseases. The screening component was calculated using the 2007/ 
2008 average value budgeted by the Regional Health Service for Pap tests. The annual budget allocated to the four-cohort strategy of vaccination and to the associated programme of screening in Basilicata was $€ 2.5$ million. As the analysis refers to an actual 12-month time period, both cost and budget values have not been discounted.

- Size of female population eligible for vaccination and inclusion (denominator of $\mathrm{x}_{1}$ ). All girls in the Basilicata region who were born in 1996, 1993, 1990 or 1983 and who were still alive in July 2007 were taken into account.

- Expected benefit of each of the two alternatives $\left(\mathrm{E}_{\mathrm{i}}\right)$. Using recently published Italian data, ${ }^{14} 18 \quad 21$ the annual number of cervical events induced by HPV, as well as the number averted by both the screening and the quadrivalent vaccine (using a three-cohort strategy of vaccination), were estimated. These cases were related to the pertinent female population of 2006 (provided by the Italian National Institute of Statistics-ISTAT $\left.{ }^{20}\right)$. The number of events was assumed to be unaffected by the inclusion of the fourth cohort (women aged 25 years). The proportion of cases of disease and of cases where disease was avoided was assessed for the whole Italian population.

The expected benefit weighted by both demographics and the frequency of disease was calculated using the following formulae:

$$
\begin{aligned}
& \mathrm{E}_{1}=1-\sum_{\mathrm{k}}\left(\frac{\mathrm{A}_{\mathrm{k}}}{\mathrm{P}} \times \frac{\mathrm{A}_{\mathrm{k}}}{\operatorname{Tot}\left(\mathrm{A}_{\mathrm{k}}\right)}\right) \times 100 \\
& \mathrm{E}_{2}=1-\sum_{\mathrm{k}}\left(\frac{\mathrm{D}_{\mathrm{k}}}{\mathrm{P}} \times \frac{\mathrm{D}_{\mathrm{k}}}{\operatorname{Tot}\left(\mathrm{D}_{\mathrm{k}}\right)}\right) \times 100,
\end{aligned}
$$

where $A_{k}$ and $D_{k}$ represent the number of women assigned to the two alternative options (screening alone or vaccination and screening in combination, respectively), $\mathrm{P}$ is the female population of Italy on 1 January 2006, and the index $\mathrm{k}$ represents the different HPVrelated diseases, such as abnormal Pap smears, precancerous cervical lesions (CIN1, CIN2-3), invasive cervical cancer and anogenital warts. The incidence of HPVrelated diseases that would occur with screening alone and with vaccination and screening corresponded to 90.77 and 36.94 per 10000 women, respectively. The equations represent the weighted expected benefit of screening alone $\left(E_{1}\right)$ and of vaccination and screening $\left(\mathrm{E}_{2}\right)$, expressed in percentage terms as the difference between the unit $(100 \%)$ and the incidence of HPVrelated diseases associated with each of the two alternative strategies. It is worth noting that screening alone has no effect on the detection of anogenital warts.

Ex-post objective: the assessment of the allocative efficiency actually achieved by the screening and the quadrivalent immunisation programmes $\mathbf{1 2}$ months after the beginning of its implementation (July 2007)

An observational and retrospective data collection was designed to assess the allocative efficiency of the two programmes. All girls in the Basilicata region who had a recorded vaccination and who belonged to one of the four selected cohorts (aged 12, 15, 18 and 25 years in 2007) were considered to be eligible for enrolment. Girls who had an incomplete record of vaccination were excluded. The collection of data began in July 2007 and continued for 18 months to ensure full compliance with the recommended schedule of vaccination $(0,2$, 6 months) within a 12-month time horizon.

\section{Sources of data}

Data on rates of screening and vaccination, allocation of budgets and costs (including the total spent on prevention) were obtained directly from each of the five participating Local Healthcare Authorities ${ }^{\mathrm{i}}$, all of which fall within the remit of the region. The data were related to the period from January 2007 to December 2008. The cost of a full course of quadrivalent vaccination (three doses) was $€ 315$, including the costs of administering the vaccine.

The regional demographic archive provided information on subjects' date of birth, sex and healthcare identification number, whereas data on the course of vaccination (including the patients' names and healthcare identification numbers, together with dates of issue, brand names and batch numbers of the vaccine) were obtained from the regional vaccination register. All personal data were replaced with a univocal numerical code, which ensured that both archives were anonymous at source (in full compliance with the Italian Privacy Law-Decree 196, 30 June 2003). The observational and retrospective design of this data collection did not necessitate the collection of informed consent from the subjects.

\section{Statistical analysis}

Differences among groups were tested using one-way analysis of variance for continuous variables, while Pearson's $\chi^{2}$ test was used for categorical variables. All the tests were two tailed, and $\mathrm{p}$ values that were $<0.05$ were considered to be statistically significant. Sensitivity analyses were performed to assess the uncertainties associated with the estimates of allocative efficiency. Specifically, we varied the cost of the quadrivalent vaccine by reducing it by $15 \%$ twice and both increased and reduced the budget by $10 \%$. This is because changes of such an order of magnitude are fairly likely to occur. The bound optimisation model and the sensitivity analyses were carried out using Mathematica, V.6.0, and the statistical analyses were performed using SPSS for Windows, V.16 (2002).

\section{RESULTS AND DISCUSSION}

The mean weighted benefit of the combined prevention strategy (vaccination and screening) resulted significantly

'The Local Health Authority is a body delegated by the Italian National Health Service to deliver local healthcare services, commonly at provincial level. 
Figure 1 Sensitivity analysis of allocative efficiency performed on the basis of variations in the main parameters implied in the bound optimisation model. Figure shows the optimal rate of coverage is highly elastic to large reductions in price of the anti-human papillomavirus (HPV) vaccine of choice, almost linearly elastic to changes in total budget and population eligible for anti-HPV prevention programmes and virtually inelastic to the cost of screening.

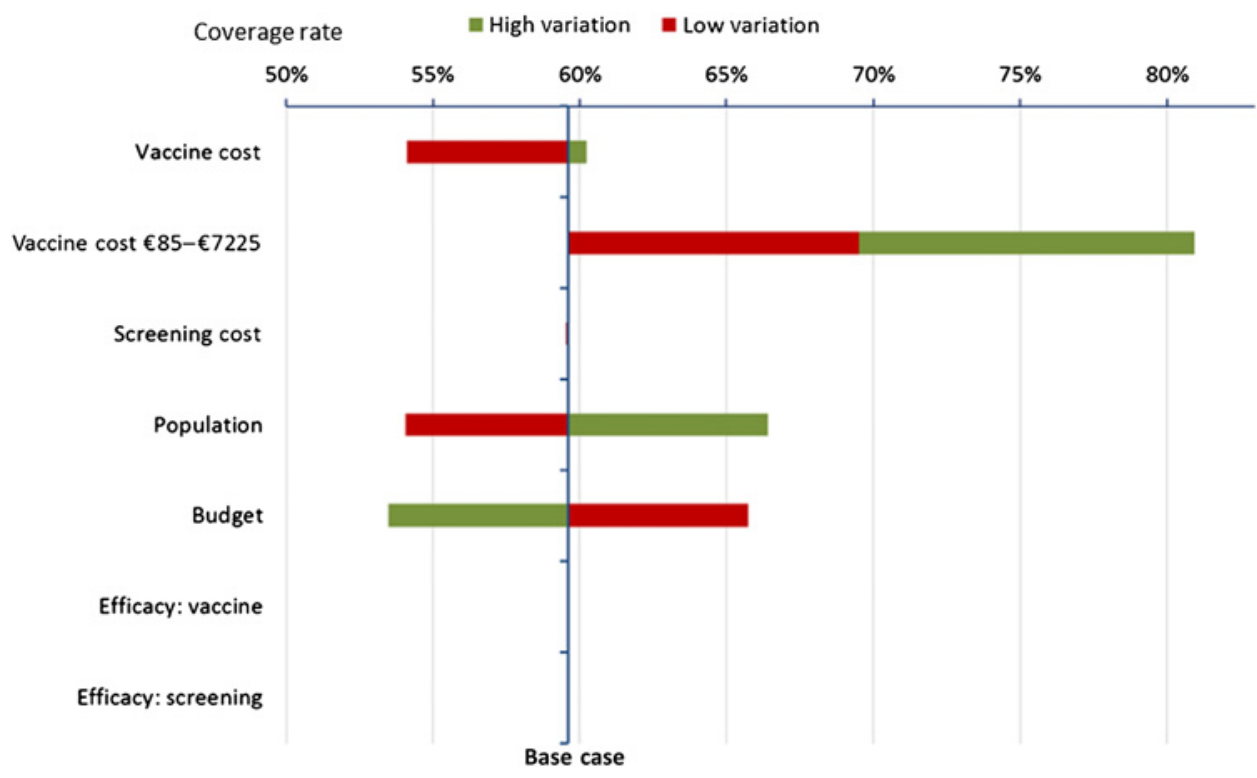

Twelve months after the beginning of the immunisation programme, the actual rate of coverage reached $72.8 \% \pm 2.0 \%$. This figure is $21 \%$ higher than the regional public health target with a difference that was statistically significant $(\mathrm{p}<0.001)$.

The highest vaccination coverage rate was reported in the cohort of girls aged 12 years $(84.7 \% \pm 2.0 \%)$. However, it should be noted that similar values were
Figure 2 Sensitivity analysis of allocative efficiency performed on the basis of variations in budget and cost of vaccine. Figure shows the general case where, by using an optimisation procedure, the authorities in Basilicata could maintain the current high rate of coverage with a reduced budget (equivalent to a reduction in the cost of the vaccine from $€ 85$, point $\alpha$, to $€ 72.5$ per dose, point $\beta$ ). Alternatively, the rate of coverage could be reduced to the optimal value of $69 \%$, thereby achieving the concomitant savings (point $\gamma$ ). Further reductions in price, as reflected in the progression towards point $\delta$, should provide scope for savings that can be represented by the shaded area $(\alpha \gamma \delta)$. In this way, regional decision makers could authorise the reallocation of resources to other programmes of prevention, while maintaining the expected high level of efficiency of the planned programme of vaccination against human papillomavirus. Regional authorities might also decide to reduce the allocated budget by $10 \%$. In such scenario, although the intercept is still within the boundary of the allocative efficiency curve, an immediate mean decrease of vaccination coverage rate of $10.1 \% \pm 0.9 \%$ would be observed. This would have the knock-on effect of reducing some of the clinical benefits. 
Figure 3 Relationship between coverage rate and number of cohorts vaccinated. ${ }^{21}$ Figure illustrates the expected long-term effect of the vaccination, correlated to the number of cohorts targeted. The combined reduction of all events, such as abnormal Pap smears, colposcopies, Atypical Squamous Cells of Undetermined Significance, Low-grade Squamous Intraepithelial Lesion, High-grade Squamous Intraepithelial Lesions, cervical cancer and genital warts, showed a significant difference between the second and the third cohort. The vaccination of three to four cohorts was likely to enhance the percentage of events avoided, shortening the time required to reach the optimal cost/effective outcome.

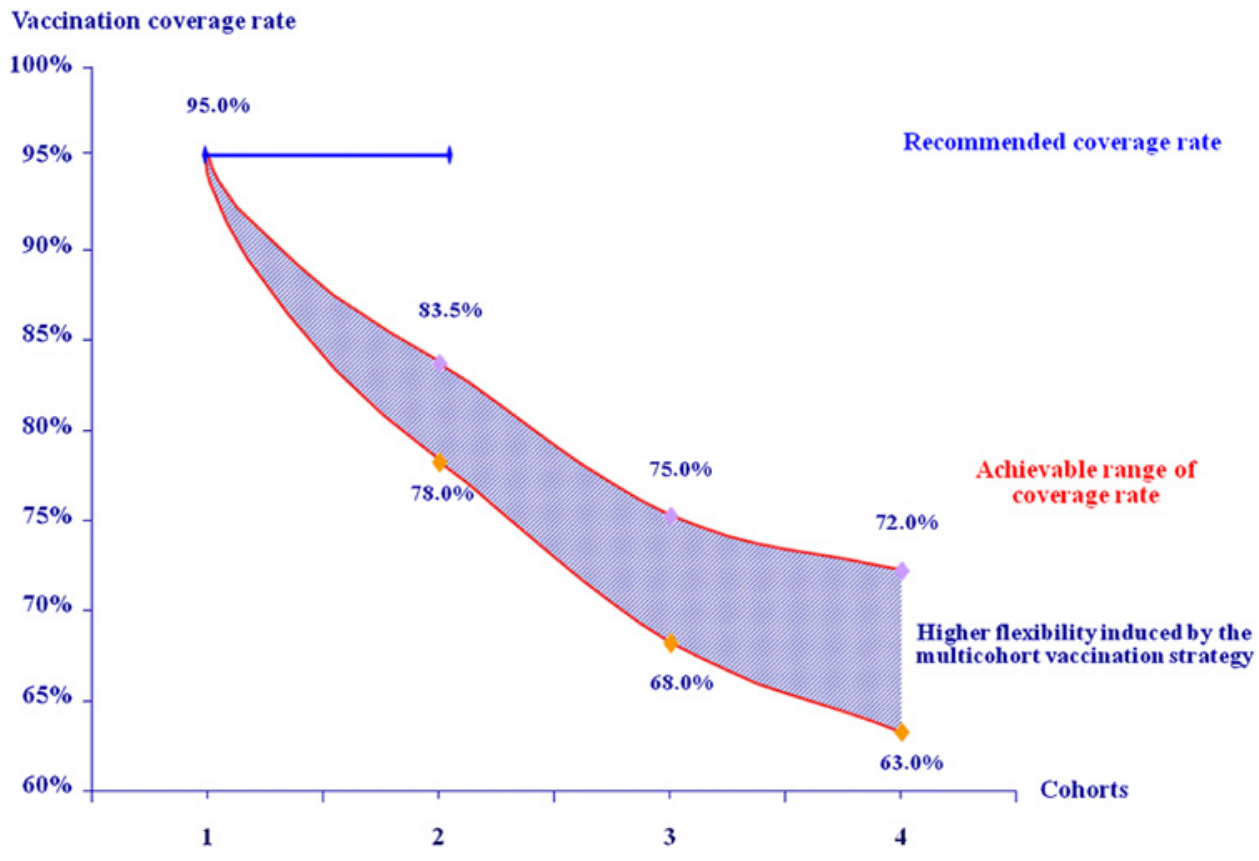

recorded in the two chronologically subsequent cohorts ( 15 and 18 years) with rates of $79.9 \% \pm 4.3 \%$ and $78.1 \% \pm$ $3.4 \%$ for girls aged 15 and 18 years, respectively. In contrast, a statistically significantly lower coverage rate $(52.1 \% \pm 3.1 \%)$ was observed in the cohort of women aged 25 years. This rate was significantly below the average value of $72.8 \%(p<0.0001)$ and was also significantly less than the value required to achieve the allocative efficiency predicted by the bound optimisation model $(\mathrm{p}<0.001)$.

Based on a sensitivity analysis performed on the bound optimisation model, figure 1 shows that the optimal rate of coverage is highly elastic to large reductions in price of the anti-HPV vaccine of choice, almost linearly elastic to changes in total budget and population eligible for anti-HPV prevention programmes and virtually inelastic to the cost of screening. This is mostly due to the inelasticity of the optimal coverage to marginal changes in the efficacy of immunisation and screening in preventing HPV-related diseases, estimated in the model as $91 \%$ and $37 \%$, respectively.

In the unlikely scenario where the screening programme resulted more effective than the anti-HPV vaccination, then the vaccination coverage would drop to $0 \%$.

The optimal rate of coverage was initially exceeded in the Basilicata region when the multicohort strategy of vaccination against HPV was used, achieving an actual inefficient allocation of resources between screening and vaccination, in favour of the second. If the price of the vaccine were to be reduced to $€ 85$ per dose, the optimal rate of vaccination coverage would increase to $69.5 \%$ (see point $\gamma$ in figure 2). As a matter of fact, in September 2008, the price paid for the quadrivalent vaccine by the Basilicata region dropped to $€ 85$ per dose.
Figure 2 shows the general case where, by using an optimisation procedure, the authorities in Basilicata could maintain the current high rate of coverage with a reduced budget (equivalent to a reduction in the cost of the vaccine from $€ 85$, point $\alpha$, to $€ 72.5$ per dose, point $\beta$ ). Alternatively, the rate of coverage could be reduced to the optimal value of $69 \%$, thereby achieving the concomitant savings (point $\gamma$ ).

Further reductions in price, as reflected in the progression towards point $\delta$, should provide scope for savings that can be represented by the shaded area $(\alpha \gamma \delta)$. In this way, regional decision makers could authorise the reallocation of resources to other programmes of prevention, while maintaining the expected high level of allocative efficiency of the budget allocated to the prevention of HPV-related pathologies.

Regional authorities might also decide to reduce the allocated budget by $10 \%$. In such scenario, although the intercept is still within the boundary of the allocative efficiency curve in figure 2 , an immediate mean decrease of vaccination coverage rate of $10.1 \% \pm 0.9 \%$ would be observed. This would have the knock-on effect of reducing some of the expected benefits.

Our findings for the 12-month period of our study demonstrate that the Basilicata region was able to leverage the reduction in the price of the vaccine from $€ 100$ to $€ 85$ per phial to achieve an higher than planned coverage rate $(73 \%$ vs $60 \%)$, progressively reducing the inefficiency of the actual resource allocation to the quadrivalent multicohort vaccination from $21 \%$ in the period July 2007 to August 2008 to an almost negligible 5\% after September 2008, when the price reduction came into effect. In the context of the Italian national programme of vaccination, the Basilicata region is in full compliance with the guidelines established by 
the Italian State-Regions Conference, which in turn were determined in accordance with both the Ministry of Health and the National Institute of Health. Moreover, the implementation of a multicohort strategy of vaccination in the region should provide clinical and economic benefits 5-8 years earlier than would be expected with a single-cohort strategy. Figure 3 provides an explanation of the benefits associated with the multicohort programme. Our findings are consistent with those of a modelling study published in $2008,{ }^{18}$ which demonstrated that the greater flexibility in the rate of coverage that results from vaccinating between three and four cohorts rather than one (in the range $63.0 \%-72.0 \%$ for a four-cohort strategy compared with a range of $78.0 \%-$ $83.5 \%$ for a two-cohort strategy) enables a higher efficiency of vaccination to be maintained. ${ }^{21}$

The present study does have some limitations. The bound optimisation model is subject to the condition that the programmes evaluated are completely divisible, with constant return to scale and that every subject included receives a fraction of the total expected benefit. Concerning the first condition, although we cannot assume that healthcare programmes are completely divisible, we can assume that there are sufficiently many interventions that each one is small in value compared with the whole: thus, the expected value is approximately a continuum. ${ }^{24}$ The return to scale of anti-HPV immunisation is unlikely to be constant due to the effect of herd immunity, ${ }^{27}$ although at present no epidemiological data are available to confirm a non-linear relationship between coverage and expected reduction of events. In essence, the effect of heard immunity on the bound optimisation model is similar to a reduction in price of the vaccine, by providing further reduction of events at the same level of vaccination coverage. Although this effect should presumably be more relevant for levels of coverage below the optimal threshold estimated by the bound optimisation level $(59.6 \%)$, the possibility to quantify the incremental reduction of HPVinduced events as a function of vaccination coverage would allow to estimate more precisely the optimal allocation of resources between immunisation and coverage. We can still assume that the return to scale of the anti-HPV immunisation is constant within the ranges taken into consideration to determine the allocative efficiency of the allowable budget. Lastly, the third condition is fully respected since every woman included in the study maintains the access to the screening programme, so she will receive a fraction of the expected benefit.

Lastly, a major limitation of the adopted model is embedded in its static and deterministic design, while a statistical dynamic approach would further improve the reliability of the outcomes.

\section{CONCLUSIONS}

The use of the Bound Optimisation Model was found to be a very useful and rational approach to the determi- nation of the optimal budget allocation given a number of ex-ante constraints as well as the ex-post, short-term assessment of the governance of resources allocated by the Basilicata region to the implementation of a multicohort programme of quadrivalent vaccination against HPV. However, the model does not resolve all the issues related to the cost-effectiveness of such programmes of vaccination. Therefore, further probabilistic models or observational studies using measured outcomes are required. These should be designed specifically to minimise the uncertainties associated with the large number of variables that need to be considered in such a complex problem.

\section{Author affiliations}

${ }^{1}$ CEIS Sanità-Centre for Health Economics and Management (CHEM), Faculty of Economics, Tor Vergata University, Rome, Italy

${ }^{2}$ Institute of Leadership and Management in Health (ILMH), Kingston University, London, UK

${ }^{3}$ Department of Epidemiology and Public Health, University College London, London, UK

${ }^{4}$ Department of Statistics, Bicocca University, Milan, Italy

${ }^{5}$ Department of Health, Safety and Social Solidarity, Personal Services and the Community, Region Basilicata, Italy

${ }^{6}$ Zurich University, Zurich, Switzerland

Acknowledgements The authors wish to acknowledge the support and relevant contributions of Professor Mike Drummond and Professor Bengt Jonsson who were also involved in the extensive and rapid peer review of this article.

Contributors FSM, GF and AC: conception and design, drafting the article and final approval of the version to be published. GB, AM, LG and GB: analysis and interpretation of data, revising it critically for important intellectual content and final approval of the version to be published. PZ, GM, GC, FL: conception and design, revising it critically for important intellectual content and final approval of the version to be published.

Funding This work was supported with an unrestricted funding from Sanofi Pasteur MSD, Italy.

Competing interests None.

Provenance and peer review Not commissioned; externally peer reviewed.

Data sharing statement There is no additional data available.

\section{REFERENCES}

1. Parkin DM, Bray F, Ferlay J, et al. Global cancer statistics, 2002. CA Cancer J Clin 2005;55:74-108.

2. Ferlay J, Bray F, Pisani P, et al. GLOBOCAN 2002: Cancer Incidence, Mortality and Prevalence Worldwide. IARC Cancer Base No. 5, Version 2.0. Lyon (France): IARC Press, 2004.

3. Ricciardi A, Largeron N, Giorgi Rossi P, et al. Incidence of invasive cervical cancer and direct costs associated with its management in Italy. Tumori 2009;95:146-52.

4. Costa S, Favato G. Evaluation of the Economic Impact Produced by the Prevention of Events Induced by the HPV 6-11Virus Types Contained in the Quadrivalent Vaccine. 2008. http://www.ssrn.com/ abstract $=1080113$ (accessed 11 Apr 2011).

5. Giorgi Rossi P, Ricciardi A, Choet C, et al. Epidemiology and costs of cervical cancer screening and cervical dysplasia in Italy. BMC Public Health 2009;9:71. http://www.biomedcentral.com/1471-2458/9/71 (accessed 11 Apr 2011).

6. Coleman D, Day N, Douglas G, et al. European guidelines for quality assurance in cervical cancer screening. Eur J Cancer 1993;29A (Suppl 4):S1-38.

7. Kulasingam SL, Myers ER. Potential health and economic impact of adding a human papillomavirus vaccine to screening programs. JAMA 2003;290:781-9.

8. Taira AV, Neukermans CP, Sanders GD. Evaluating human papillomavirus vaccination programs. Emerg Infect Dis 2004;10:1915-23. 
9. Goldie SJ, Kholi M, Grima D, et al. Projected clinical benefits and cost-effectiveness of a human papillomavirus $16 / 18$ vaccine. $J$ Natl Cancer Inst 2004;96:604-15.

10. Barnabas RV, Laukkanen $P$, Koskela $P$, et al. Epidemiology of HPV 16 and cervical cancer in Finland and the potential impact of vaccination: mathematical modelling analyses. PLoS Med 2006;3:624-32.

11. Elbasha EH, Dasbach EJ, Insinga RP. Model for assessing human papillomavirus vaccination strategy. Emerg Infect Dis 2007;13:28-41.

12. Dasbach E, Insinga R, Elbasha E. The epidemiological and economic impact of a quadrivalent human papillomavirus vaccine $(6 / 11 / 16 / 18)$ in the UK. BJOG 2008. http://onlinelibrary.wiley.com/doi/10.1111/ j.1471-0528.2008.01743.x/pdf (accessed 11 Apr 2011).

13. Chesson HW, Ekwueme DU, Saraiya M, et al. Cost-effectiveness of human papillomavirus vaccination in the United States. Emerg Infect Dis 2008;14:244-51.

14. Mennini FS, Giorgi Rossi P, Palazzo F, et al. Health and economic impact associated with a quadrivalent HPV vaccine in Italy. Gynecol Oncol 2009;112:370-6.

15. Capri S, Bamfi F, Marocco A, et al. Impatto clinico ed economico della vaccinazione anti-HPV (clinical and economic impact of anti-HPV vaccination). Ital J Public Health 2007;4(2 Suppl 1): S36-54.

16. Rawlins MD, Culyer AJ. National Institute for Clinical Excellence and its value judgments. BMJ 2004;329:224-7.

17. Devlin N, Parkin D. Does NICE a cost-effectiveness threshold and what other factors influence its decisions? A binary choice analysis. Health Econ 2004;13:437-52.
18. Favato G, Pieri V, Mills R. Cost/Effective Analysis of Anti-HPV Vaccination Programme in Italy: A Multi-Cohort Markov Model. 2007 http://www.ssrn.com/abstract=961847 (accessed 11 Apr 2011).

19. Cicchetti A, Ruggeri M, Mennini FS, et al. Analisi economica pe l'estensione della vaccinazione contro l'influenza: risparmi sociali e analisi di Budget Impact. Pharmacoeconomics Italian Research Articles 2008;10:137-50. http://adisonline.com/pecitalian/Abstract 2008/10030/Analisi_economica_per_I_estensione_della.2.aspx (accessed 2 Mar 2012).

20. Istituto nazionale di statistica (ISTAT). Popolazione Residente per età, sesso e stato civile al $1^{\circ}$ gennaio 2006. http://demo.istat.it/ pop2006/index.html (accessed 11 Apr 2011).

21. Mennini FS, Costa S, Favato G, et al. Anti-HPV vaccination: a review of recent economic data for Italy. Vaccine 2009;27:A54-61.

22. The Italian Ministry of Health. Principles and Objectives of the Ministry of health. http://www.ministerosalute.it/ministero/ sezMinistero.jsp?label=principi (accessed 11 Apr 2011).

23. Palmer S, Torgerson DJ. Definitions of efficiency. BMJ 1999;318:1136

24. Weinstein $M$, Zeckhauser R. Critical ratios and efficient allocation. $J$ Public Econ 1973;2:147-57.

25. Garber AM, Phelps CE. Economic foundations of cost-effectiveness analysis. J Health Econ 1997;16:1-31.

26. Cicchetti A, Ruggeri M, Gitto L, et al. Extending influenza vaccination to individuals aged 50-64: a budget impact analysis. Int J Technol Assess Health Care 2010;26:288-93.

27. Jit M, Chapman R, Hughes $\mathrm{O}$, et al. Comparing bivalent and quadrivalent human papillomavirus vaccines: economic evaluation based on transmission model. BMJ 2011;343.5775. 